Research article

\title{
Basal cytokeratins in breast tumours among BRCA1, BRCA2 and mutation-negative breast cancer families
}

\author{
Hannaleena Eerola1,2, Mira Heinonen ${ }^{3,4}$, Päivi Heikkilä5 ${ }^{5}$ Outi Kilpivaara², Anitta Tamminen², \\ Kristiina Aittomäki ${ }^{6}$, Carl Blomqvist ${ }^{1}$, Ari Ristimäki3,4 and Heli Nevanlinna ${ }^{2}$
}

\begin{abstract}
1Department of Oncology, Helsinki University Central Hospital, Haartmaninkatu, 00029 HUS, Helsinki Finland
2Department of Obstetrics and Gynecology, Helsinki University Central Hospital, Haartmaninkatu, 00029 HUS, Helsinki, Finland

${ }^{3}$ Department of Pathology/HUSLAB and Haartman Institute, Helsinki University Central Hospital, Haartmaninkatu,00029 HUS, Helsinki, Finland

${ }^{4}$ Genome-Scale Biology Program, Biomedicum Helsinki, University of Helsinki, Haartmaninkatu, 00014 Helsinki, Finland

${ }^{5}$ Department of Pathology, Helsinki University Central Hospital, Haartmaninkatu,00029 HUS, Helsinki, Finland

${ }^{6}$ Department of Clinical Genetics, Helsinki University Central Hospital, Haartmaninkatu,00029 HUS, Helsinki, Finland
\end{abstract}

Corresponding author: Hannaleena Eerola, hannaleena.eerola@fimnet.fi

Received: 5 Jul 2007 Revisions requested: 24 Aug 2007 Revisions received: 23 Nov 2007 Accepted: 14 Feb 2008 Published: 14 Feb 2008

Breast Cancer Research 2008, 10:R17 (doi:10.1186/bcr1863)

This article is online at: http://breast-cancer-research.com/content/10/1/R17

(c) 2008 Eerola et al.; licensee BioMed Central Ltd.

This is an open access article distributed under the terms of the Creative Commons Attribution License (http://creativecommons.org/licenses/by/2.0), which permits unrestricted use, distribution, and reproduction in any medium, provided the original work is properly cited.

\begin{abstract}
Introduction Finding new immunohistochemical markers that are specific to hereditary breast cancer could help us to select candidates for BRCA1/BRCA2 mutation testing and to understand the biological pathways of tumour development.

Methods Using breast cancer tumour microarrays, immunohistochemical expression of cytokeratin (CK)-5/6, CK14 and CK-17 was evaluated in breast tumours from BRCA1 families $(n=46)$, BRCA2 families $(n=40)$, non-BRCA1/ BRCA2 families $(n=358)$ and familial breast cancer patients with one first-degree relative affected by breast or ovarian cancer $(n=270)$, as well as from patients with sporadic breast cancer $(n=364)$. Staining for CK-5/6, CK-14 and CK-17 was compared between these groups and correlated with other clinical and histological factors.
\end{abstract}

Results CK-5/6, CK-14 and CK-17 were detected mostly among oestrogen receptor (ER)-negative, progesterone receptor (PR)-negative and high-grade tumours. We found the highest percentages of samples positive for these CKs among ER-negative/HER2-negative tumours. In univariate analysis, CK14 was significantly associated with tumours from $B R C A 1$ (39\%; $P<0.0005)$, BRCA2 (27\%; $P=0.011)$, and non$B R C A 1 / B R C A 2(21 \% ; P<0.005)$ families, as compared with sporadic tumours (10\%). However, in multivariate analysis, CKs were not found to be independently associated with $B R C A 1$ or $B R C A 2$ mutation status, and the most effective predictors of $B R C A 1$ mutations were age at onset, HER2 status, and either ER or PR status.

Conclusion Although our study confirms that basal CKs can help to identify BRCA1 mutation carriers, this effect was weaker than previously suggested and CKs did not independently predict $B R C A 1$ mutation either from sporadic or familial breast cancer cases. The most effective, independent predictors of BRCA1 mutations were age at onset, HER2 status, and either ER or PR status, as compared with sporadic or non-BRCA1/ BRCA2 cancers.

\section{Introduction}

Human breast cancers form a heterogeneous group of cancers. There are many subclassifications, and attempts have been made to identify differences between and characterize such subgroups. Earlier immunohistochemical studies, and recently gene expression studies as well, have been conducted to classify tumours [1]. One of the subgroups proposed is the so-called basal-like group of tumours, which are associated with breast cancers that express neither oestrogen receptor (ER) nor human epidermal growth factor receptor (HER) 2, but express frequently 'basal' cytokeratins (CKs) such as CK-5/6, CK-14 and CK-17. These CKs are intermediate filaments that are found in the cells of the basal layer of normal breast ducts. Therefore, the term 'basal' is generally used, although the origin of breast tumour cells expressing these CKs is unknown. Moreover, the definition of basal phenotype

$\overline{\mathrm{Cl}}=$ confidence interval; $\mathrm{CK}=$ cytokeratin; $\mathrm{ER}=$ oestrogen receptor; $\mathrm{HER}=$ human epidermal growth factor receptor; $\mathrm{OR}=$ odds ratio; $\mathrm{PR}=$ progesterone receptor; TMA $=$ tissue microarray. 
in breast tumours is conflicting; for example, not all tumours classified as basal-like by microaray analysis express basal CKs [2]. In general, these tumours are associated with high grade and stage [3], but conflicting findings have been reported regarding the independent prognostic significance of the basal phenotype [4]. Although basal CK expression may predict early relapse among nonselected tumours, the clinical outcome of basal tumours has been found to be similar to that of nonbasal ER-negative tumours [5].

The general features of basal phenotype tumours are the same as those of tumours in BRCA1 mutation carriers [1]. In particular, expression of CK-5/6 [6,7] and CK-5/14 [8] has been associated with BRCA1 tumours. Moreover, Lakhani and colleagues [9] tested five basal markers (CK-14, CK-5/6, CK-17, epidermal growth factor and osteonectin), all of which were significantly associated with BRCA1 tumours as compared with unselected breast tumours.

In addition to the CKs, it has been clear for some time that $B R C A 1$-associated tumours have distinct pathological features when compared with sporadic cancers. BRCA1 tumours have higher tumour grade, less ER and progesterone receptor (PR) expression, less HER2 expression and overexpression of $\mathrm{p53}$, and a higher proportion of medullary than sporadic cancers [10]. Furthermore, they exhibit high Ki-67index and over-express proteins such as cyclin-E, cyclin- $A$ and cyclin- $\mathrm{B}_{1}[10]$. The pathological phenotype of $B R C A 2$-associated tumours is inconsistent, and in many cases no significant differences have been found between BRCA2-associated and sporadic cancers [11-13]. However, some studies have found BRCA2-associated tumours to be negative for HER2 and to over-express CHEK2 (checkpoint kinase 2) and RAD51 (homolog of Saccharomyces cerevisiae Rad51) [10]. The basal CK frequencies have been found to be similar in this group of tumours and sporadic ones [9].

There is much need for markers that could be used to distinguish patients and families who are likely to carry a BRCA1/ BRCA2 germline mutation from mutation-negative families and from breast cancer patients in general. Such markers could facilitate targeting of expensive and time-consuming genetic testing to individuals who are most likely to carry those mutations. It is also necessary to learn more about the biological characteristics of these tumours in order to foster development of less radical prevention strategies than risk-reducing surgery and to identify possible targets for drug development. It has been suggested that CK-5/6, CK-14, P-cadherin and epidermal growth factor receptor may have utility as a first-line immunohistochemical test for the presence of germline BRCA1 mutation [14]. In this study, we evaluated expression of basal CKs among tumours from BRCA1, BRCA2 and non$B R C A 1 / B R C A 2$ families (families with several breast cancer patients without $B R C A 1$ or BRCA2 mutations), and sporadic breast cancer patients. It is important to compare tumours of mutation carriers with tumours from families including several cancer cases, and not just to compared them with sporadic tumours, because this is the situation usually encountered in the clinical setting of genetic counselling.

\section{Materials and methods \\ Breast cancer patients and tumours}

A series of 884 consecutive, newly diagnosed breast cancer patients was recruited from the Department of Oncology (Helsinki University Central Hospital) during the period from 1997 to 1998 and during the year 2000, as described in detail by Syrjäkoski and coworkers [15] and Kilpivaara and colleagues [16]. Familial breast cancer patients $(n=546)$ were recruited from the Departments of Oncology and Clinical Genetics, as described by Eerola and coworkers [17]. This series includes breast cancer families with at least three first-degree or second-degree relatives with breast or ovarian cancer, as well as patients with one first-degree relative affected by breast or ovarian cancer, with no restriction on age. All of the breast cancer families and unselected patients were tested for BRCA1 and BRCA2 mutations by mutation analysis of the entire coding sequences and exon/intron boundaries of the genes using protein truncation test and denaturing gradient gel electrophoresis, or as previously described [18-20], except for 158 patients from families with two affected members, in whom $B R C A 1$ and BRCA2 mutation status was not determined.

We collected all available paraffin blocks containing enough tumour tissue from primary breast cancers of unselected and familial breast cancer patients, and the most representative area of the tumour was punched to produce a breast cancer tissue microarray (TMA), including two to four cores (diameter $0.6 \mathrm{~mm}$ ) from each original block, as partially described previously [21]. Altogether, the TMAs included 1,335 invasive breast cancer tumours; 921 of these were from familial patients (577 from families with three or more first-degree or second-degree relatives with breast or ovarian cancer, including the proband, and 344 from families with two affected firstdegree relatives) and 414 from sporadic patients (patients without the above-defined family history of breast or ovarian cancer). Fifty-two of the tumours from familial patients were from patients of families positive for BRCA1 mutation and 56 tumours were from patients of families positive for BRCA2 mutation.

In this study we excluded lobular carcinomas as ambiguous, as well as tumours from noncarriers in the BRCA1/BRCA2 families and tumour from patients for whom no representative tumour tissue was available on the TMA arrays. Finally, the staining findings was obtained for a total of 1,078 tumours, which included 622 cancers from the unselected breast cancer patient series. Combined with the family material, CK expression was evaluated in the following: 46 cancers from $B R C A 1$ families; 40 cancers from BRCA2 families; 358 cancers from non-BRCA1/2 families; 270 cancers from patients 
with one first-degree or second-degree relative affected by breast or ovarian cancer; and 364 cancers from patients with sporadic breast cancer (patients with no family history of breast cancer).

We studied haematoxylin and eosin stained sections of the original blocks for histological diagnosis and grading (by the same pathologist $[\mathrm{PH}]$ ) Grading was performed using the Scarff-Bloom-Richardson method, as modified by Elston and Ellis [22]. All of the TMA slides were stained with antibodies to CK-5/6, CK-14 and CK-17. Briefly, $5 \mu \mathrm{m}$ sections were cut from paraffin-embedded blocks, deparafinated in xylene and dehydrated in a series of graded alcohols. Sections that were immnunostained with antibodies against CK-5/6 (dilution 1:25; Dako, Glostrup, Denmark) and CK-14 (dilution 1:20; Novocastra, Newcastle upon Tyne, UK) were first pretreated in a microwave oven in Tris-EDTA ( $\mathrm{pH}$ 9.0), incubated with the primary antibodies for 30 minutes and visualized using the Envision detection system (Dako) and LabVision autostainer (Thermo Fisher Scientific Inc., Fremont, CA, USA). Sections that were immunostained with antibodies against CK-17 (dilution 1:20; Vector Laboratories, Burlingame, CA, USA) were first pretreated in a microwave oven in $10 \mathrm{mmol} / \mathrm{l}$ citric acid (pH 6.0), incubated with primary antibodies for 30 minutes and visualized using (Renaissance) TSA-Biotin system NEL 700 kit (PerkinElmer, Massachusetts, USA). Specimens were considered negative if immunopositivity was found in $0 \%$ to $10 \%$ and positive if $11 \%$ to $100 \%$ of the cancer cells exhibited immunopositivity. A cut-off value of $>10 \%$ results in clearly positive staining.

All the TMA slides were stained using routine methodology with p53 antibodies (1:300; Dako, Copenhagen, Denmark; described previously by Eerola and coworkers [21]). Samples were considered positive when $20 \%$ of the cancer cells stained positive. Amplification of the HER2 oncogene was determined by chromogenic in situ hybridization (as described by Tanner and coworkers [23]); none to five replications was considered negative, and more than six replications was considered positive. Information on ER and PR status was acquired from pathology reports. The staining methodology and evaluation of the results are applied routinely in breast cancer diagnostics and are previously described by Eerola and coworkers [21]. Samples were considered positive when $10 \%$ of the cancer cells stained positive. The information on stage was acquired from patient records.

\section{Statistical analysis}

Statistical analysis was conducted using SPSS for Windows (SPSS Inc., Chigaco, IL, USA). We tested the differences in dichotomous variables by $\chi^{2}$ test or Fisher's exact test, and multivariate analysis was conducted using logistic regression (backward conditional, $P$ for stepwise removal 0.05). All $P$ values are two sided.

\section{Ethics}

The study was performed with informed consent from the patients as well as with approval from the Ethics Committee (E8) of the Helsinki University Central Hospital and from the Ministry of Social Affairs and Health in Finland.

\section{Results}

Tumours expressing basal CKs (CK-5/6, CK-14 and CK-17) were more likely to be ER negative and PR negative, and to occur in younger breast cancer patients. CK-5/6 expression was also significantly associated with high grade and p53 positivity, and expression of CK-5/6 and CK-14 with HER2 negativity. CK14 was significantly associated with BRCA1 mutation but also with BRCA2 and non-BRCA1/BRCA2 groups of cancers, as compared with sporadic breast cancers (Table 1). We found all three CK markers to be highly significantly associated with the subgroup of tumours negative for both ER and HER2. Within this subgroup of cancers, CK-5/6 was positive in $33 \%$ of 167 cancers $(P<0.0005)$, CK-14 in $35 \%$ of 161 cancers $(P<0.0005)$, and CK-17 in $10 \%$ of 158 cancers $(P=0.001)$, as compared with $9 \%, 11 \%$ and $4 \%$, respectively, among the tumours in which one or both of the markers (ER of HER2) was positive.

$B R C A 1$ associated cancers were more often high grade, ER negative, PR negative, p53 positive and HER2 negative, as well as CK 14 positive, as compared with all other groups (Table 2). CK-5/6 frequency was also higher but not significantly so if compared with non-BRCA1/BRCA2 or sporadic cancers. However, in multivariate analysis, including all factors from Table 2, neither CK-5/6 nor CK-14 were independent predictors of BRCA1 mutations. After stepwise removal of markers that were not significant at the $5 \%$ level, independent predictors were found to be age at onset, HER2 negativity and ER and/or PR status, depending on the analysis. In the analysis comparing BRCA1 tumours with sporadic tumours, the factors in the final model were ER (for negative status: odds ratio $[O R]=11.2,95 \%$ confidence interval $[\mathrm{Cl}]=4.3$ to 29.2 ), HER2 (for negative status: $\mathrm{OR}=4607.4,95 \% \mathrm{Cl}=0$ to 3.8 $\times 10^{21}$ ) and age at onset (for age $<50$ years: $O R=4.3,95 \%$ $\mathrm{Cl}=1.6$ to 11.2 ). Compared with non-BRCA1/BRCA2 families the final model included PR (for negative status: OR = 10.0, 95\% Cl=3.5 to 28.6), HER2 (for negative status: OR $=4309.7,95 \% \mathrm{Cl}=0$ to $3.3 \times 10^{24}$ ) and age at onset (for age $<50$ years: $\mathrm{OR}=5.0,95 \% \mathrm{Cl}=1.9$ to 13.1 ).

BRCA2-associated tumours were also significantly more often negative for ER, PR and HER2, and detected at earlier age than sporadic tumours or non-BRCA1/BRCA2 tumours. CK14 was expressed more frequently than among sporadic cancers. However, in multivariate analysis, including all significant variables from univariate analysis, the only independent predictors of $B R C A 2$ mutation were the age at onset under 50 years (OR $=3.7,95 \% \mathrm{Cl}=1.5$ to 8.7 ), PR negativity (OR $=2.9$, $95 \% \mathrm{Cl}=1.2$ to 6.9 ) and HER2 negativity (OR $=3705.2$, 
Table 1

Association of CK-5/6, CK-14 and CK-17 with clinicopathological features

\begin{tabular}{|c|c|c|c|c|c|c|c|c|c|c|c|}
\hline & \multirow[t]{2}{*}{ All } & \multirow[t]{2}{*}{$\%$} & \multicolumn{3}{|c|}{ CK-5/6 } & \multicolumn{3}{|c|}{ CK-14 } & \multicolumn{3}{|c|}{ CK-17 } \\
\hline & & & Positive/alla & $\%$ & $P$ & Positive/alla & $\%$ & $P$ & Positive/alla & $\%$ & $P$ \\
\hline All & 1078 & 100 & $129 / 982$ & 13.1 & & $151 / 955$ & 15.8 & & $41 / 931$ & 4.4 & \\
\hline \multicolumn{12}{|l|}{ Oestrogen receptor } \\
\hline Negative & 266 & 26.0 & $67 / 241$ & 27.8 & $<0.0005$ & $63 / 236$ & 26.7 & $<0.0005$ & $20 / 236$ & 8.5 & 0.001 \\
\hline Positive & 757 & 74.0 & $56 / 692$ & 8.1 & & $78 / 671$ & 11.6 & & $21 / 650$ & 3.2 & \\
\hline \multicolumn{12}{|l|}{ Progesterone receptor } \\
\hline Negative & 388 & 38.0 & $74 / 353$ & 21.0 & $<0.0005$ & $76 / 348$ & 21.8 & $<0.0005$ & $26 / 346$ & 7.5 & $<0.001$ \\
\hline Positive & 633 & 62.0 & $49 / 578$ & 8.5 & & $65 / 557$ & 11.7 & & $15 / 538$ & 2.8 & \\
\hline \multicolumn{12}{|l|}{ Grade } \\
\hline 1 & 231 & 21.6 & $17 / 194$ & 8.8 & $<0.0005$ & $32 / 193$ & 16.6 & 0.097 & $3 / 185$ & 1.6 & 0.021 \\
\hline ॥ & 453 & 42.5 & $43 / 420$ & 10.2 & & $52 / 403$ & 12.9 & & $15 / 391$ & 3.8 & \\
\hline III & 383 & 35.9 & $69 / 359$ & 19.2 & & $65 / 350$ & 18.6 & & $23 / 347$ & 6.6 & \\
\hline \multicolumn{12}{|l|}{$\mathrm{T}$} \\
\hline 1 & 649 & 61.3 & $69 / 580$ & 11.9 & 0.159 & $89 / 556$ & 16.0 & 0.790 & $27 / 549$ & 4.9 & 0.434 \\
\hline 2 to 4 & 409 & 38.7 & $58 / 386$ & 15.0 & & $59 / 384$ & 15.4 & & $14 / 366$ & 3.8 & \\
\hline \multicolumn{12}{|l|}{$N$} \\
\hline 0 & 596 & 56.6 & $72 / 538$ & 13.4 & 0.607 & $85 / 524$ & 16.2 & 0.477 & $23 / 506$ & 4.5 & 0.659 \\
\hline 1 & 457 & 43.4 & $52 / 424$ & 12.3 & & $60 / 413$ & 14.5 & & $16 / 405$ & 4.0 & \\
\hline \multicolumn{12}{|l|}{$M$} \\
\hline 0 & 997 & 96.5 & $122 / 910$ & 13.4 & 0.882 & $144 / 885$ & 16.3 & 0.269 & $39 / 861$ & 4.5 & 0.706 \\
\hline 1 & 36 & 3.5 & $4 / 32$ & 12.5 & & 3/33 & 9.1 & & $1 / 32$ & 3.1 & \\
\hline \multicolumn{12}{|l|}{ p53 } \\
\hline Negative & 772 & 76.5 & $76 / 732$ & 10.4 & $<0.0005$ & $108 / 718$ & 15.0 & 0.264 & $27 / 687$ & 3.9 & 0.120 \\
\hline Positive & 237 & 23.5 & $51 / 230$ & 22.2 & & $40 / 220$ & 18.2 & & $14 / 217$ & 6.5 & \\
\hline \multicolumn{12}{|l|}{ HER2 } \\
\hline Negative & 774 & 84.9 & $107 / 756$ & 14.2 & 0.058 & $132 / 740$ & 17.8 & $<0.0005$ & $34 / 709$ & 5.7 & 0.764 \\
\hline Positive & 138 & 15.1 & $11 / 135$ & 9.3 & & $7 / 133$ & 5.3 & & $5 / 120$ & 4.2 & \\
\hline \multicolumn{12}{|l|}{ Age (years) } \\
\hline$\geq 50$ & 668 & 62.0 & $65 / 603$ & 10.8 & 0.006 & $80 / 580$ & 13.8 & 0.033 & $19 / 570$ & 3.3 & 0.045 \\
\hline$<50$ & 410 & 38.0 & $64 / 379$ & 16.9 & & $71 / 375$ & 18.9 & & $22 / 361$ & 6.1 & \\
\hline$B R C A 1$ & 46 & 4.3 & $6 / 34$ & 17.6 & $0.250^{b}$ & $13 / 33$ & 39.4 & $<0.0005^{b}$ & $1 / 43$ & 2.3 & $0.866^{b}$ \\
\hline BRCA2 & 40 & 3.7 & $2 / 26$ & 7.7 & $0.599 b$ & $7 / 26$ & 26.9 & $0.011^{b}$ & $0 / 34$ & 0 & $0.326^{b}$ \\
\hline Non-BRCA1/BRCA2 & 358 & 33.2 & $44 / 333$ & 13.2 & $0.383^{b}$ & $67 / 324$ & 20.7 & $<0.0005^{b}$ & $11 / 320$ & 3.4 & $0.624^{b}$ \\
\hline Two affected & 270 & 25.0 & $40 / 253$ & 15.8 & $0.087^{b}$ & $30 / 244$ & 12.3 & $0.469^{b}$ & $20 / 209$ & 9.6 & $0.001^{b}$ \\
\hline Only sporadic & 364 & 33.8 & 37/336 & 11.0 & Ref. & $34 / 328$ & 10.4 & Ref. & $9 / 325$ & 2.8 & Ref. \\
\hline
\end{tabular}

a Positive staining/samples available. bCompared with 'only sporadic'. CK, cytokeratin; HER, human epidermal growth factor receptor. 
Table 2

\begin{tabular}{|c|c|c|c|c|c|c|c|c|c|c|c|c|c|c|}
\hline & \multicolumn{3}{|c|}{$B R C A 1$ cases } & \multicolumn{3}{|c|}{$B R C A 2$ cases } & \multicolumn{3}{|c|}{ Non-BRCA1/BRCA2 } & \multicolumn{3}{|c|}{ Two affected } & \multicolumn{2}{|c|}{ Sporadic } \\
\hline & $n$ & $\%$ & $P$ & $n$ & $\%$ & $P$ & $n$ & $\%$ & $P$ & $n$ & $\%$ & $P$ & $n$ & $\%$ \\
\hline All & 46 & & & 40 & & & 358 & & & 270 & & & 364 & \\
\hline \multicolumn{15}{|c|}{ Oestrogen receptor } \\
\hline Negative & 33 & 75.0 & $<0.0005$ & 15 & 38.5 & 0.013 & 79 & 24.3 & 0.294 & 64 & 24.9 & 0.248 & 75 & 20.9 \\
\hline Positive & 11 & 25.0 & & 24 & 61.5 & & 246 & 75.7 & & 193 & 75.1 & & 283 & 79.1 \\
\hline \multicolumn{15}{|c|}{ Progesterone receptor } \\
\hline Negative & 37 & 84.1 & $<0.0005$ & 21 & 53.8 & 0.014 & 115 & 35.6 & 0.657 & 93 & 36.3 & 0.548 & 122 & 34.0 \\
\hline Positive & 7 & 15.9 & & 18 & 46.2 & & 208 & 64.4 & & 163 & 63.7 & & 237 & 66.0 \\
\hline \multicolumn{15}{|l|}{ Grade } \\
\hline 1 & 1 & 2.2 & $<0.0005$ & 8 & 20.0 & 0.933 & 82 & 23.5 & 0.717 & 59 & 21.9 & 0.974 & 81 & 22.3 \\
\hline$\|$ & 11 & 24.4 & & 17 & 42.5 & & 154 & 44.1 & & 117 & 43.3 & & 154 & 42.4 \\
\hline III & 33 & 73.3 & & 15 & 37.5 & & 113 & 32.4 & & 94 & 34.8 & & 128 & 35.3 \\
\hline \multicolumn{15}{|c|}{$\mathrm{T}$} \\
\hline 1 & 21 & 46.7 & 0.079 & 18 & 54.5 & 0.516 & 229 & 65.6 & 0.144 & 162 & 60.4 & 0.976 & 219 & 60.3 \\
\hline 2 to 4 & 24 & 53.3 & & 15 & 45.5 & & 120 & 34.4 & & 106 & 39.6 & & 144 & 39.7 \\
\hline \multicolumn{15}{|l|}{$N$} \\
\hline 0 & 32 & 71.1 & 0.026 & 15 & 44.1 & 0.289 & 214 & 61.1 & 0.043 & 142 & 53.8 & 0.965 & 193 & 53.6 \\
\hline 1 & 13 & 28.9 & & 19 & 55.9 & & 136 & 38.9 & & 122 & 46.2 & & 167 & 46.4 \\
\hline \multicolumn{15}{|l|}{ M } \\
\hline 0 & 41 & 93.2 & 0.570 & 28 & 90.3 & 0.245 & 338 & 98.8 & 0.005 & 255 & 96.6 & 0.386 & 335 & 95.2 \\
\hline 1 & 3 & 6.8 & & 3 & 9.7 & & 4 & 1.2 & & 9 & 3.4 & & 17 & 4.8 \\
\hline \multicolumn{15}{|l|}{ p53 } \\
\hline Negative & 25 & 62.5 & 0.017 & 26 & 76.5 & 0.707 & 261 & 76.5 & 0.399 & 193 & 75.1 & 0.233 & 267 & 79.2 \\
\hline Positive & 15 & 37.5 & & 8 & 23.5 & & 80 & 23.5 & & 64 & 24.9 & & 70 & 20.8 \\
\hline \multicolumn{15}{|l|}{ HER2 } \\
\hline Negative & 30 & 100 & 0.017 & 27 & 100 & 0.023 & 256 & 85.3 & 0.598 & 192 & 82.1 & 0.587 & 269 & 83.8 \\
\hline Positive & 0 & 0 & & 0 & 0 & & 44 & 14.7 & & 42 & 17.9 & & 52 & 16.2 \\
\hline \multicolumn{15}{|l|}{ CK-5/6 } \\
\hline Negative & 28 & 82.4 & 0.250 & 24 & 92.3 & 0.599 & 289 & 86.8 & 0.383 & 213 & 84.2 & 0.087 & 299 & 89.0 \\
\hline Positive & 6 & 17.6 & & 2 & 7.7 & & 44 & 13.2 & & 40 & 15.8 & & 37 & 11.0 \\
\hline \multicolumn{15}{|l|}{ CK-14 } \\
\hline Negative & 20 & 60.6 & $<0.0005$ & 19 & 73.1 & 0.011 & 257 & 79.3 & $<0.0005$ & 214 & 87.7 & 0.469 & 294 & 89.6 \\
\hline Positive & 13 & 39.4 & & 7 & 26.9 & & 67 & 20.7 & & 30 & 12.3 & & 34 & 10.4 \\
\hline \multicolumn{15}{|l|}{ CK-17 } \\
\hline Negative & 42 & 97.7 & 0.866 & 34 & 100 & 0.326 & 309 & 96.6 & 0.624 & 189 & 90.4 & 0.001 & 316 & 97.2 \\
\hline Positive & 1 & 2.3 & & 0 & 0 & & 11 & 3.4 & & 20 & 9.6 & & 9 & 2.8 \\
\hline \multicolumn{15}{|l|}{ Age (years) } \\
\hline$\geq 50$ & 16 & 34.8 & $<0.0005$ & 22 & 55.0 & 0.004 & 215 & 60.1 & 0.035 & 97 & 35.9 & 0.356 & 246 & 67.6 \\
\hline$<50$ & 30 & 65.2 & & 18 & 45.0 & & 143 & 39.9 & & 173 & 64.1 & & 118 & 32.4 \\
\hline
\end{tabular}

CK, cytokeratin; HER2, human epidermal growth factor receptor 2. 
$95 \% \mathrm{Cl}=0$ to $\left.2.5 \times 10^{22}\right)$, as compared with sporadic tumours.

Tumours from familial non-BRCA1/BRCA2 patients were similar to sporadic ones, although they were of lower stage and CK-14 was expressed more often than among sporadic tumours (Table 2). Age at onset was slightly younger than among patients with sporadic breast cancer (median age at onset: 54 and 57 years; $P=0.001$ ). Tumours from families with two affected members did not differ from sporadic tumours.

\section{Discussion}

In this study expression of CK-5/6, CK-14 and CK-17 was found to be strongly associated with the subgroup of tumours negative for ER and HER2, as suggested previously for the basal subgroup of breast tumours [3]. However, although our study confirms that basal CKs, especially CK-14, can help to identify BRCA1 mutation carriers, this effect was weaker than in previous studies.

Because of differences in antibodies, staining and tissue processing procedures in different studies, it is difficult to compare findings between studies directly. For example, we chose a cut-off point $(>10 \%)$ for CKs that results in clearly positive staining. Previously, rather different cut-off points had been used, from any positivity to $20 \%$. However, the frequencies in the present study among unselected breast cancer patients correlate with the previous literature, in which about $2 \%$ to $19 \%$ of unselected breast cancers were positive for CK-5, CK-6 and CK-14 [7-9,24,25]. Moreover, the subgroups in our study are comparable because the material was collected uniformly from the same hospital, and microarray tissue blocks enable staining of all samples at the same time and under the same conditions. Highly significant correlations between findings obtained using this kind of multicore system and of whole sections of the original blocks have also been demonstrated $[26,27]$.

Similarly to previous studies, we found that expression of any one or all of the markers CK-5/6, CK-14 and CK-17 correlates with ER negativity $[4,8,24]$, PR negativity [8], high grade $[4,8,24,25]$, early age at diagnosis $[4,24,25]$ and p53 positivity [4]. Numerous studies have reported that basal CKs are more frequently positive in BRCA1 tumours than in sporadic breast cancers [6-9]. In our study as well, CK-14 was significantly associated with BRCA1 carrier status, and CK-5/6 frequency was also higher than among sporadic breast cancers but the difference was not statistically significant. In the study conducted Lakhani and coworkers [9], 57.6\% of the BRCA1associated tumours stained positive for CK-5/6 and $60.6 \%$ for CK-14. Corresponding figures in our study were $17.6 \%$ and $39.4 \%$, although the percentages in control populations in both studies were similar (Table 2).
In the study conducted by Foulkes and colleagues [6], CK-5/ 6 was detected in 56\% of the 72 ER/HER2-negative cancers, and the frequency was even higher (88\%) among the 17 BRCA1 mutation carriers with ER/HER2-negative cancers. In our study CK-5/6 positivity was detected in a large proportion (33\%) of the $167 \mathrm{ER} / \mathrm{HER} 2-$ negative cancers as well, but only in $25 \%$ of the $20 \mathrm{ER} / \mathrm{HER} 2$-negative and BRCA1-related cancers. CK-14 was detected in 35\% of 161 ER/HER2-negative cancers and $37 \%$ of the ER/HER2-negative and BRCA1-positive group.

Few studies have used multivariate analysis to address the significance of basal CKs in predicting BRCA1 mutations. In the study conducted by Lakhani and colleagues [9], CK-14, CK$5 / 6$, CK-17, osteonectin, epidermal growth factor receptor, $\mathrm{ER}$ and grade were included in a multiple regression model. They found that independent markers for BRCA1 positivity were $\mathrm{ER}, \mathrm{CK}-5 / 6$ and $\mathrm{CK}-14$. In our multivariate analysis, including all factors significant in univariate analysis, the studied basal CKs were not independent predictors of BRCA1 mutation status. However, in the multivariate analysis with only basal CKs together with ER and grade, as in the study by Lakhani and colleagues [9], the remaining significant factors were CK-14 (OR = 3.3, 95\% Cl=1.4 to 7.9$)$ and $\mathrm{ER}(\mathrm{OR}=$ $6.2,95 \% \mathrm{Cl}=2.7$ to 14.2 ). Thus, the effect of $\mathrm{CK}-14$ was similar to that detected by Lakhani and colleagues. However, the CK-5/6 result was different from their study, because it was not significant in univariate or multivariate analysis. One factor that may affect this is the strong association of CK-5/6 with age at diagnosis, as shown in Table 1 . The tumours included in our study were unselected for age, and about 35\% of the $B R C A 1$ tumours in our study were diagnosed at age 50 years or older. We previously showed that tumours of $B R C A 1$ and $B R C A 2$ carriers aged 50 years or older differ significantly from those of younger patients [28]. However, CK-14 expression correlates strongly with the HER2 status, as well as with ER and PR status, and if we included all of these studied variables in the logistic regression analysis, then the remaining independent factors for BRCA1 mutation, in the final model, were only age at onset, HER2 negativity and ER and/or PR status, depending on the analysis. Age at onset, HER2 and PR were not included in multivariate analysis conducted by Lakhani and colleagues [9].

We also detected CK-14-positive staining among $27 \%$ of $B R C A 2$ associated tumours and $21 \%$ of the non-BRCA1/ $B R C A 2$ associated tumours. These proportions are significantly higher than the $10 \%$ among the sporadic tumours $(P=$ 0.011 and $P<0.0005$, respectively). This finding could be influenced by chance in a low population frequency with multiple testing, or by the younger age in these subgroups compared with the older patients with sporadic breast cancer, because CK expression also correlates with tumours occurring at earlier ages. In multivariate analysis, CKs were not independently associated with BRCA2 mutation status. To 
exclude the possibility of missed $B R C A 1$ mutations among non-BRCA1/BRCA2 families, we conducted the same analysis after excluding families with ovarian cancer cases, but this did not alter the results. In the study conducted by Lakhani and colleagues [9], none of the basal markers was significantly associated with BRCA2 status. However, in their study as well, the frequencies of CK-5/6 (15\%) and CK-14 (24\%) were higher among BRCA2 cases than among controls $(7 \%$ and $12 \%)$.

\section{Conclusion}

ER and PR strongly correlate with each other (Pearson's R = $0.657, P<0.0005$ in this study), and both of them were found to be effective in predicting BRCA1 mutation. However, although our study confirms that basal CKs can help to identify $B R C A 1$ mutation carriers, this effect was weaker than previously suggested and CKs did not independently predict $B R C A 1$ mutation either in sporadic or familial breast cancer cases. One factor that may affect this is the strong association of CK-5/6 with age at diagnosis, because the tumours included in our study were unselected for age and about 35\% of the BRCA1 tumours in our study were diagnosed at age 50 years or older. In our material CKs also correlated strongly with many other tumour characteristics, such as ER negativity, PR negativity, high grade, p53 positivity, HER2 negativity and age at onset. If we consider all factors analyzed, the most effective independent predictors of $B R C A 1$ mutations were age at onset, HER2 status and either ER or PR status. The same factors appear also to predict $B R C A 2$ mutations, although not as strongly as $B R C A 1$ mutations.

\section{Competing interests}

The authors declare that they have no competing interests.

\section{Authors' contributions}

All authors were involved in critically revising the manuscript and gave their approval for the manuscript to be published. Furthermore, HE participated in patient and tumour sample collection, performed statistical analysis and drafted the manuscript. $\mathrm{MH}$ and $\mathrm{AR}$ scored and interpreted the immunoassays. PH studied the original tumour samples for histological diagnosis and grading, and organized immunoassays. OK participated in designing and coordination of the study and helped to draft the manuscript. AT carried out molecular genetic studies. KA participated in patient collection and was responsible for genetic counselling of patients. CB participated in organizing patient and tumour collections. $\mathrm{HN}$ participated in the study design and coordination, and helped to draft the manuscript.

\section{Acknowledgements}

We wish to thank the families who participated in this study; Minna Merikivi and Nina Puolakka for their help in sample collection; and the Finnish Cancer Registry for cancer diagnosis and identification numbers for archival material of the pathology laboratories. Grants: Academy of Finland (110663), Clinical Research Fund of Helsinki University Central
Hospital, Finnish Breast Cancer Group, Finnish-Norwegian Medical Foundation, The Finnish Cancer Society, Maud Kuistila Foundation, and Sigrid Juselius Foundation.

\section{References}

1. Sorlie T, Tibshirani R, Parker J, Hastie T, Marron JS, Nobel A, Deng $\mathrm{S}$, Johnsen H, Pesich R, Geisler S, Demeter J, Perou CM, Lonning PE, Brown PO, Borresen-Dale A, Botstein D: Repeated observation of breast tumour subtypes in independent gene expression data sets. Proc Natl Acad Sci USA 2003, 100:8418-8423.

2. Livasy CA, Karaca G, Nanda R, Tretiakova MS, Olopade O, Moore $D$, Perou CM: Phenotypic evaluation of the basal-like subtype of invasive breast carcinoma. Mod Patho/ 2006, 19:264-271.

3. Gusterson B, Ross DT, Heath VJ, Stein T: Basal cytokeratins and their relationship to the cellular origin and functional classification of breast cancer. Breast Cancer Res 2005, 7:143-148.

4. Foulkes W, Brunet J-S, Stefansson IM, Straume O, Chappuis PO, Begin LR, Hamel N, Goffin JR, Wong N, Trudel M, Kapusta L, Porter $\mathrm{P}$, Akslen LA: The prognostic implication of the basal-like (Cyclin E high/p27 low/p53+/Glomeruloid-microvascularproliferation+) phenotype of BRCA1-related breast cancer. Cancer Res 2004, 64:830-835.

5. Jumppanen M, Gruvberger-Saal S, Kauraniemi $P$, Tanner M, Bendahl P-O, Lundin M, Krogh M, Kataja P, Borg A, Fernö M, Isola J: Basal-like phenotype is not associated with patient survival in estrogen-reseptor-negative breast cancers. Breast Cancer Res 2007, 9:R16.

6. Foulkes W, Stefansson IM, Chappuis PO, Begin LR, Goffin JR, Wong N, Trudel M, Akslen LA: Germline BRCA1 mutations and basal epithelial phenotype in breast cancer. J Natl Cancer Inst 2003, 95:1482-1485.

7. Palacios J, Honrado E, Osorio A, Diez O, Rivas C: Re: Germline BRCA1 mutations and basal epithelial phenotype in breast cancer. J Natl Cancer Inst 2004, 96:712-714.

8. Laakso M, Loman N, Borg A, Isola J: Cytokeratin 5/14-positive breast cancer: true basal phenotype confined to BRCA1 tumors. Mod Pathol 2005, 18:1321-1328.

9. Lakhani SR, Reis-Filho JS, Fulford L, Penault-Llorca F, Van der Vijver M, Parry S, Bishop T, Benitez J, Rivas C, Bignon Y, ChanClaude J, Hamann U, Cornelisse C, Devilee P, Beckmann M, Nestle-Krämling C, Daly PA, Haites N, Varley JM, Lalloo F, Evans C, Maugard C, Meijers-Heijboer H, Klijn JGM, Olah E, Gusterson B, Pilotti S, Radice P, Scherneck S, Sobol H, et al:: Prediction of BRCA1 status in patients with breast cancer using estrogen receptor and basal phenotype. Clin Cancer Res 2005, 11:5175-5180.

10. Honrado E, Osorio A, Palacios J, Benitez J: Pathology and gene expression of hereditary breast tumours associated with BRCA1, BRCA2 and CHEK2 gene mutations. Oncogene 2006, 25:5837-5845.

11. Phillips $\mathrm{K}$, Andrulis IL, Goodwin PJ: Breast carcinomas arising in carriers of mutations in BRCA1 or BRCA2: are they prognostically different? J Clin Oncol 1999, 17:3653-3663.

12. Noguchi S, Kasugai T, Miki Y, Fukutomi T, Emi M, Nomizu T: Clinicopathologic analysis of BRCA1 - or BRCA2 associated hereditary breast carcinoma in Japanese Women. Cancer 1999, 85:2200-22205.

13. Lakhani SR, van de Vijver MJ, Jacquemier J, Anderson TJ, Osin PP, McGuffog L, Easton D: The pathology of familial breast cancer: predictive value of immunohistochemical markers estrogen receptor, progesterone receptor, HER-2, and p53 in patients with mutations in BRCA1 and BRCA2. J Clin Oncol 2002, 20:2310-2318.

14. Foulkes W, Akslen LA: Re: Germline BRCA1 mutations and basal epithelial phenotype in breast cancer. I Nat/ Cancer Inst 2004, 96:714.

15. Syrjäkoski $K$, Vahteristo $P$, Eerola $H$, Tamminen $A$, Kivinummi $K$, Sarantaus L, Holli K, Blomqvist C, Kallioniemi O, Kainu T, Nevanlinna $\mathrm{H}$ : Population-based study of BRCA1 and BRCA2 mutations in 1035 unselected Finnish breast cancer patients. J Nat/ Cancer Inst 2000, 92:1529-1531.

16. Kilpivaara $\mathrm{O}$, Bartkowa J, Eerola $\mathrm{H}$, Syrjäkoski $\mathrm{K}$, Vahteristo $P$, lukas J, Blomqvist C, Holli K, Heikkilä P, Sauter G, Kallioniemi O-P, Bartek J, Nevanlinna $\mathrm{H}$ : Correlation of CHEK2 protein expression and c.1100delC mutation status with tumor characteris- 
tics among unselected breast cancer patients. Int $\mathrm{J}$ Cancer 2005, 113:575-580.

17. Eerola $H$, Blomqvist $C$, Pukkala $E$, Pyrhönen $S$, Nevanlinna $H$ : Familial breast cancer in southern Finland: how prevalent are breast cancer families and can we trust the family history reported by patients? Eur J Cancer 2000, 36:1143-1148.

18. Vehmanen $P$, Friedman LS, Eerola $H$, Sarantaus L, Pyrhönen S, Ponder BAJ, Muhonen T, Nevanlinna $\mathrm{H}$ : A low proportion of BRCA2 mutations in Finnish Breast cancer families. Am J Hum Genet 1997, 60:1050-1058.

19. Vehmanen $\mathrm{P}$, Friedman LS, Eerola H, McClure M, Ward B, Sarantaus L, Kainu T, Syrjäkoski K, Pyrhönen S, Kallioniemi O, Muhonen T, Luce M, Frank TS, Nevanlinna H: Low proportion of BRCA1 and BRCA2 mutations in Finnish breast cancer families: evidence for additional susceptibility genes. Hum Mol Genet 1997, 6:2309-2315.

20. Vahteristo $P$, Eerola $H$, Tamminen $A$, Blomqvist $C$, Nevanlinna $H: A$ probability model for predicting BRCA1 and BRCA2 mutations in breast and breast-ovarian cancer families. $\mathrm{Br} J$ Cancer 2001, 84:704-708.

21. Eerola $\mathrm{H}$, Heikkilä $\mathrm{P}$, Tamminen A, Aittomäki $\mathrm{K}$, Blomqvist $\mathrm{C}$, Nevanlinna $\mathrm{H}$ : Histopathological features of breast tumours in BRCA1, BRCA2, and in mutation negative breast cancer families. Breast Cancer Res 2005, 7:R93-R100.

22. Elston C, Ellis I: Pathological prognostic factors in breast cancer. I. The value of histological grade in breast cancer: experience from a large study with long-term follow-up. Histopathology 1991, 19:403-410.

23. Tanner M, Gancberg D, Di Leo A, Larsimont D, Rouas G, Piccart MJ: Chromogenic in situ hybridization: a practical alternative for fluorescence in situ hybridization to detect HER-2/neu oncogene amplification in archival breast cancer samples. $A m$ J Pathol 2000, 157:1467-1472.

24. Abd El-Rehim DM, Pinder SE, Paish CE, Bell J, Blamey RW, Robertson JFR, Nicholson RI, Ellis I: Expression of luminal and basal cytokeratins in human breast carcinoma. J Pathol 2004, 203:661-671.

25. Ribeiro-Silva A, Ramalho LNZ, Garcia SB, Brandao DF, Chahud F Zucoloto S: p53 correlates with both BRCA1 and cytokeratin 5 in invasive breast cancinomas: further evidence for the pathogenesis of the basal phenotype of breast cancer. Histopathology 2005, 47:458-466.

26. Kononen J, Bubendorf $L$, Kallioniemi $A$, Bärlund $M$, Schraml $P$, Leighton S, Torhorst J, Mihatch MJ, Sauter G, Kallioniemi O: Tissue microarrays for high-throughput molecular profiling of tumor specimens. Nat Med 1998, 4:844-847.

27. Gillet CE, Springall RJ, Barnes DM, Hanby AM: Multiple tissue core arrays in histopathology research: a validation study. J Pathol 2000, 192:549-553.

28. Eerola H, Heikkilä P, Tamminen A, Aittomäki K, Blomqvist C, Nevanlinna H: Relationship of patients' age to histopathological features of breast tumours in BRCA1, BRCA2, and in mutation negative breast cancer families. Breast Cancer Res 2005, 7:R465-R469. 